\title{
Modified graphene as novel lubricating additive with high dispersion stability in oil
}

\author{
Pu WU ${ }^{1}$, Xinchun CHEN ${ }^{1}$, Chenhui ZHANG ${ }^{1, *}$, Jiping ZHANG ${ }^{2}$, Jianbin LUO $^{1}$, Jiyang ZHANG ${ }^{3, *}$ \\ ${ }^{1}$ State Key Laboratory of Tribology, Tsinghua University, Beijing 100084, China \\ ${ }^{2}$ Tianjin Research Institute for Advanced Equipment, Tsinghua University, Tianjin 300300, China \\ ${ }^{3}$ Beijing Key Laboratory of Long-life Technology of Precise Rotation and Transmission Mechanisms, Beijing Institute of Control \\ Engineering, Beijing 100094, China
}

Received: 15 July 2019 / Revised: 15 September 2019 / Accepted: 20 September 2019

(C) The author(s) 2019.

\begin{abstract}
Graphene is a promising material as a lubricant additive for reducing friction and wear. Here, a dispersing method which combines chemical modification of graphene by octadecylamine and dicyclohexylcarbodiimide with a kind of effective dispersant has been successfully developed to achieve the remarkable dispersion stability of graphene in base oil. The stable dispersion time of modified graphene (0.5 wt\%) with dispersant ( $1 \mathrm{wt} \%)$ in PAO-6 could be up to about 120 days, which was the longest time reported so far. At the same time, the lubricant exhibits a significant improvement of tribological performance for a steel ball to plate tribo-system with a normal load of $2 \mathrm{~N}$. The coefficient of friction between sliding surfaces was $\sim 0.10$ and the depth of wear track on plate was $\sim 21 \mathrm{~nm}$, which decreased by about $44 \%$ and $90 \%$ when compared to pure PAO- 6 , respectively. Furthermore, the analysis of the lubricating mechanisms in regard to the sliding-induced formation of nanostructured tribo-film has been contacted by using Raman spectra and TEM.
\end{abstract}

Keywords: modified graphene; dispersity; lubricant additives; tribo-film

\section{Introduction}

Graphene has attracted a great deal of attention in recent years because of its unique structure and remarkable mechanical, electrical, optical and thermal properties [1-5]. In particular, graphene has been proved as an excellent candidate for both solid lubricant and lubricant additive owing to its excellent mechanical strength, low shear strength, high thermal and chemical stability, atomically smooth surface, notable specific surface area, and ultrathin film thickness [6-10].

Friction and wear can lead to energy wastage, material losses and shorter life of moving components in mechanical processes. It is reported that the direct losses from tribological contacts are closed to $23 \%$ of total fuel energy lost [11]. In order to save energy as well as to protect the mechanical components, lubrication has been demonstrated as an effective method to reduce friction and wear in multiple industrial applications. Liquid lubricant is one kind of versatile lubricant which has a wide range of uses, and graphene also has gained large interest in recent years as lubricating additives due to their low shear resistance between the layer structure, suitable size to enter the contact areas and good thermal conductivity to dissipate the heat generated during friction process [12-23].

So far, a number of studies have been conducted on the tribological behavior and lubrication mechanism of graphene as lubricating additives. Especially, it is highly necessary to ensure stable dispersion of graphene as lubricating additive in various base lubricants. One of the most effective ways to improve the dispersion stability of graphene is to regulate its physical form.

* Corresponding authors: Chenhui ZHANG, E-mail: chzhang@tsinghua.edu.cn; Jiyang ZHANG, E-mail: zjy_wsy@sina.com.cn 
Li et al. [16] prepared highly exfoliated reduced graphene oxide by thermal reduction to increase the specific surface area of graphene, and the stable dispersion time of this graphene in PAO- 6 could reach 4 days. And by adding $0.5 \mathrm{wt} \%$ reduced graphite oxide in poly ( $\alpha$-olefin) lubricant oil (PAO-6), the friction coefficient and the depth of wear track decreased by $44 \%$ and $90 \%$, respectively. Dou et al. reported a novel crumpled graphene balls as effective lubricant additives in PAO-4 [17]. It could be found that the crumpled graphene balls had better dispersion stability in base oil than graphite, carbon black and commercial reduced graphite oxide. And it was believed that graphene balls could provide ball bearing effect during the friction process, thus improving the friction-reducing and anti-wear properties of PAO-4.

Another commonly used method to improve the dispersibility of graphene is chemical modification. In order to increase the dispersion stability of graphene in water, oxidation methods are employed to graft the oxygen-containing functional group on graphene sheets. Kinoshita et al. [18] investigated the lubrication performance of graphene oxide (GO) when it was added into pure water, and they found that the friction coefficient of water with GO at a concentration of $1 \mathrm{wt} \%$ was as low as 0.05 with mild wear on tribo-pairs after friction tests. Lin et al. [19] used stearic and oleic acids as raw materials to graft alkylene chains on graphene sheets by cyclic heating method, thus improving the dispersion stability of graphene in base oil. It was found that the unmodified graphene was heavily agglomerated in base oil in 200 minutes while little precipitation could be observed for modified graphene. And the friction coefficient of base oil with $0.075 \mathrm{wt} \%$ modified graphene was about 0.12 , which was $33.3 \%$ lower than that of pure base oil.

In addition, the use of dispersants is also a feasible means to effectively improve the dispersion stability of graphene in base lubricants. For example, Cai et al. [20] used Span-80 $\left(\mathrm{C}_{24} \mathrm{H}_{44} \mathrm{O}_{6}\right)$ as dispersant to enhance the dispersibility of graphene in PAO-4. As for PAO-4 with $0.05 \mathrm{wt} \%$ graphene and $1 \mathrm{wt} \%$ Span- 80 , the suspension was darker and more turbid than PAO- 4 with $0.05 \mathrm{wt} \%$ graphene after 3 days, which demonstrated that the Span-80 could effectively improve the dispersion stability of graphene in base oil. However, the longest reported stable dispersion time of graphene in oil-based lubricants is only $\sim 30$ days so far. And there still need a lot of efforts in the development of effectively-dispersed solutions of graphene in oil to achieve longer stable dispersion time, thus meeting the imperative demands in practical applications.

Herein, a novel dispersing method is developed to achieve remarkable dispersibility of graphene as lubricant additive in PAO-6. The graphene sheets are modified with octadecylamine through a chemical synthesis process, and an effective dispersant mainly consisting of polyisobutylene succinimide is also added into the lubricant to attain enhanced graphene dispersion. The synergistic effect of chemical modification of graphene and the use of dispersant is quite impressive in terms of the improvement in dispersion stability of lubricating additive. Moreover, the tribological performance of the lubricant is also improved significantly when compared with pure PAO-6. Besides, the effect of additive concentration and the lubrication mechanism were also studied. In a word, the long-term dispersion stability of graphene in lube oil and the high-performance tribological behaviors of the lubricant have great potential to meet practical application needs.

\section{Experimental}

\subsection{Preparation and characterization of material}

GO was prepared via a modified Hummer's method while the graphite powder was used as raw material [21]. The typical preparation process of GO is as follows. Firstly, sulfuric acid was poured into a beaker in an ice bath, and then $2 \mathrm{~g}$ graphite powder and $1.6 \mathrm{~g}$ sodium nitrate were added into $140 \mathrm{~mL}$ sulfuric acid (98 wt\%), respectively. The next step was to slowly add $12 \mathrm{~g}$ of potassium permanganate to the solution while ensuring that the solution temperature was lower than $15{ }^{\circ} \mathrm{C}$ during this process. Then the beaker was placed in a fume hood for 8 hours under magnetic stirring while the temperature of the solution needed to be kept at $35^{\circ} \mathrm{C}$. Then the deionized water was gradually added into the mixture and a dark-brown suspension was obtained. After this step, the $10 \mathrm{~mL}$ hydrogen peroxide 
solution (30\%) was added into the suspension, achieving a brilliant yellow color solution. The solution was centrifuged and the product was washed with hydrogen chloride solution and deionized water. At last, the obtained solid matter was placed in a vacuum oven and dried at $60^{\circ} \mathrm{C}$ for 8 hours.

The modified graphene $(\mathrm{MG})$ was then prepared on the basis of GO. The dried GO (0.1 g) was dispersed in deionized water by ultrasonication and mechanical stirring for 30 minutes. Then octadecylamine $(0.5 \mathrm{~g})$ and dicyclohexylcarbodiimide $(0.1 \mathrm{~g})$ were added to the GO aqueous solution. The mixture was refluxed under the continuous stirring for 12 hours at $120^{\circ} \mathrm{C}$. After the reaction, it could be observed that the supernatant solution in the round bottom flask became clear, and a black solid substance was obtained at the bottom of the flask. The obtained black product was then washed 5 times by deionized water and ethanol, respectively. Finally, the modified graphene was dried in vacuum drying chamber.

Morphology and chemical structural properties of GO and MG were investigated through atomic force microscope (AFM), X-ray photoelectron spectroscopy (XPS) and Fourier transform infrared spectroscopy (FTIR).

In addition, the dispersant employed in this work was mainly composed of polyisobutylene succinimide. And the polyisobutylene succinimide was prepared with polyisobutylene, maleic anhydride and polyamine by synthetic reactions.

\subsection{Tribological measurements}

MG and dispersant were dispersed in PAO-6 by mechanical stirring for $0.5 \mathrm{~h}$ and ultrasonication for another $0.5 \mathrm{~h}$, respectively. The tribological properties of the lubricant were studied by a reciprocating tribotester (UMT-5, Brucker) with a ball-on-disk mode. The plates used in tests were bearing steels (AISI 52100) and the counter balls were also bearing steels (AISI 52100 ) with $4 \mathrm{~mm}$ diameter. The mean roughness of the balls was $20 \mathrm{~nm}$, and the surface of the plates has been polished to a roughness of $5 \mathrm{~nm}$. Balls and plates were cleaned by acetone and ethyl alcohol in ultrasonic cleaner for $10 \mathrm{~min}$ before the frictional experiments, respectively. The applied normal load during the experiments was $2 \mathrm{~N}$, resulting in an initial maximum
Hertzian contact pressure of about 1.1 GPa. Besides, the average sliding speed was $5 \mathrm{~mm} / \mathrm{s}$ under reciprocating motion. And the time for each experiment is 900 seconds. All of the friction experiments were conducted in atmospheric environment.

After the tests, the analysis of contact areas was conducted by scanning electron microscopy (SEM), Raman spectroscopy, three dimensional profilometer and transmission electron microscope (TEM).

\section{Results and discussion}

The morphologies and structures of GO and MG were analyzed by several methods. Figures 1(a) and 1 (b) show the dimensions of the prepared GO and MG detected by AFM, and it could be seen that the two-dimensional sizes of GO and MG were 2-5 $\mu \mathrm{m}$ and the thicknesses of GO and MG flakes were about 2.6 and $3.1 \mathrm{~nm}$, respectively. FTIR spectroscopy was also used in order to confirm the existence of alkyl chains. Figure 1(c) is the FTIR result for GO. The peaks appeared at $1,735,1,624,1,381$, and $1,073 \mathrm{~cm}^{-1}$ represent the $\mathrm{C}=\mathrm{O}, \mathrm{C}=\mathrm{C}, \mathrm{C}-\mathrm{O}$, and $\mathrm{C}-\mathrm{O}-\mathrm{C}$, respectively, which are typical peaks of $\mathrm{GO}$ sample and have also been reported in previous studies [22]. As for MG sample (Fig. 1(d)), the typical peaks of C-H could be observed at 2,914 and $2,846 \mathrm{~cm}^{-1}$, the peaks appeared at 1,457 and $1,368 \mathrm{~cm}^{-1}$ represent $\mathrm{CH}_{3}$, and the typical peak of $\left.\left(\mathrm{CH}_{2}\right)_{n}\right)$ is at $710 \mathrm{~cm}^{-1}$, these results confirm the existence of long alkyl chains on graphene. Besides, Figs. 1(e)-1(h) show the XPS scan spectra of GO and MG. The C/O atomic percentage of GO and MG was 68.3/30.6 and 89.4/10.2, respectively (Figs. 1(e) and 1(f)). It was believed that the conspicuous increase in carbon atomic percentages for MG is owing to the alkyl chains existed on its surface. Moreover, Figs. 1(g) and 1(h) present the spectra of C1s for GO and MG. The C1s spectra show the presences of $\mathrm{C}-\mathrm{C}$ at $284.7 \mathrm{eV}$, $\mathrm{C}-\mathrm{O}$ at $286.6 \mathrm{eV}$, and $\mathrm{C}=\mathrm{O}$ at $288.1 \mathrm{eV}$.

Dispersion stability of solid additives is quite important for an effective lubricant. Figures 2(a)-2(c) show the photos of MG and MG/dispersant in PAO-6 as a function of time. The MG $(0.5 \mathrm{wt} \%)$ and $\mathrm{MG}$ $(0.5 \mathrm{wt} \%) /$ dispersant $(1.0 \mathrm{wt} \%)$ were added in base oil with mechanical stir for $30 \mathrm{~min}$ and sonication for $60 \mathrm{~min}$, respectively, thus producing stable dispersed 
(a)

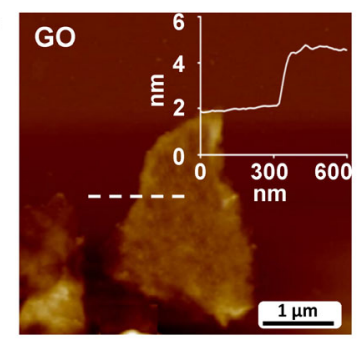

(c)
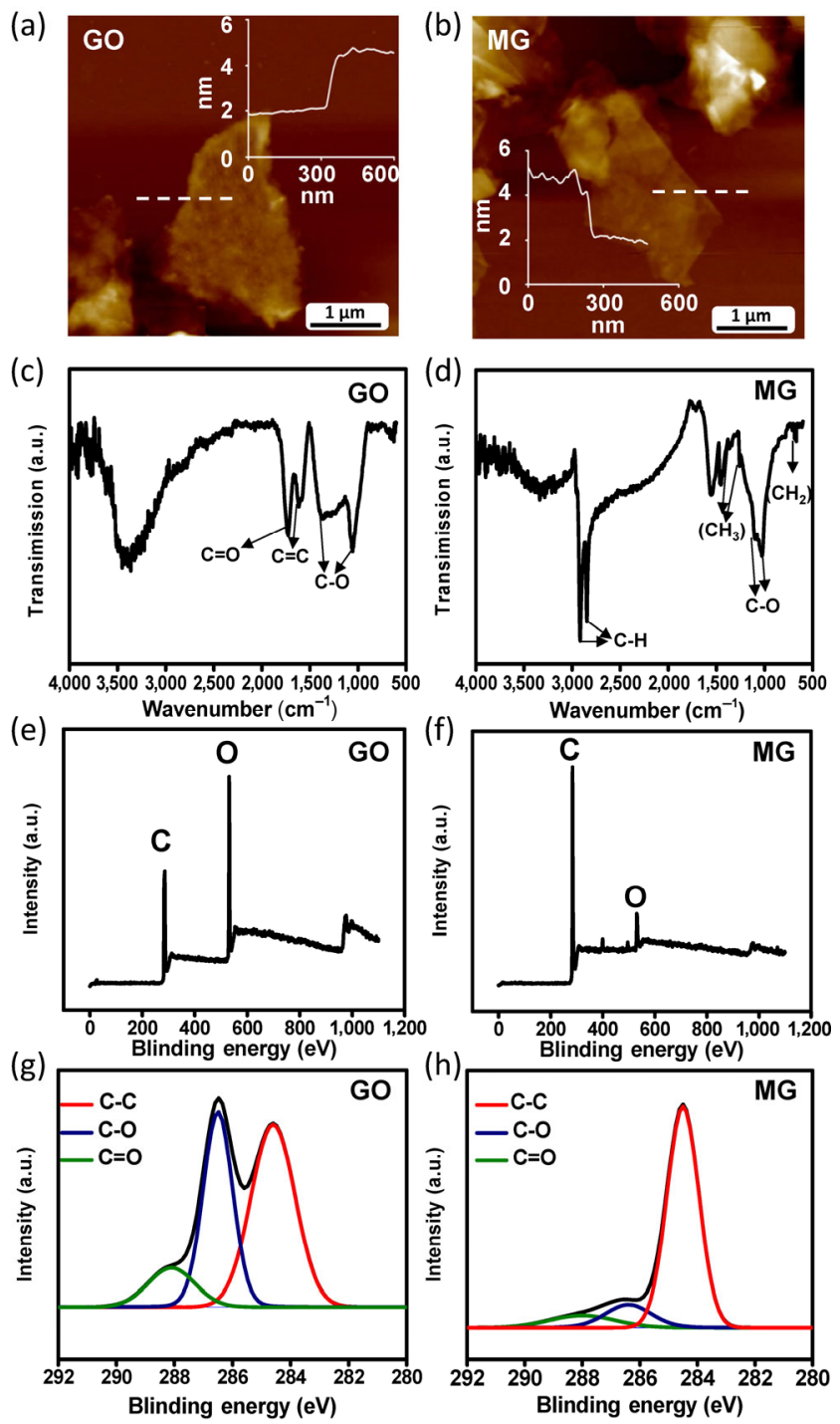

Fig. 1 AFM images of (a) GO and (b) MG, FTIR spectra of (c) GO and (d) MG, XPS survey spectra of (e) GO and (f) MG, XPS C1s spectra of (g) GO and (h) MG.

solutions. It could be observed that the stable dispersion time of MG and MG/dispersant in PAO-6 was 30 days and 120 days, respectively. Moreover, Fig. 2(e) presents the stable dispersion time of graphene reported in the literatures so far when oil was used as the base lubricant [12-14, 20, 23-28]. It could be seen that 120 days obtained in our work were the longest time. And the detailed comparison of these literatures was presented in Table S1 in the Electronic Supplementary Material (ESM). In addition, the variations of stable dispersion time of MG $(0.5 \mathrm{wt} \%)$ with different concentrations of dispersant were also studied, which was showed in Fig. S1 in the ESM. Besides, as indicated by Fig. S2 in the ESM, the viscosity of PAO-6 with MG/dispersant was $53.1 \mathrm{mPa} \cdot \mathrm{s}$ (measured by a Rheometer), which was slightly larger than that of PAO-6 with MG (49.4 mPa.s) and pure PAO-6 (46.2 $\mathrm{mPa} \cdot \mathrm{s})$.

It is well known that the solute composed of polar molecules is easily dissolved in a solvent composed of polar molecules, while the non-polar solutes are more soluble in non-polar solvents. Owing to the presence of various polar functionalities like hydroxyl and carboxyl groups, GO is hydrophilic and could be well dispersed in water, but has poor dispersibility in oil. However, many engineering applications have great demands for good dispersion stability of graphene in oil. Here, MG was functionalized with alkylamines to facilitate its well dispersion in PAO-6, and the stable dispersion time could reach $\sim 30$ days. It is believed that the interaction between alkyl chains grafted in the graphene nano-sheets and the base oil plays an important role in improving the dispersing performance [29]. Besides, the addition of dispersant could further enhance the dispersion stability of MG in PAO-6, which increased the dispersion time from $\sim 30$ to $\sim 120$ days. This phenomenon is considered to be due to the fact that the dispersant also contains long alkyl chains, which could be intertwined with the alkyl chain on MG to enhance its dispersion properties.

The tribological properties of PAO-6 with MG were analyzed by UMT-5. The applied normal load was $2 \mathrm{~N}$ and the sliding speed was $5 \mathrm{~mm} / \mathrm{s}$. Figure 3(a) shows the schematic diagram of frictional experiments. Figure 3(b) presents the friction coefficient for PAO-6 with different MG concentrations. As the content of MG gradually increased, the friction coefficient decreased first and then increased. It could be observed that the lowest friction coefficient was about 0.12 when the amount of MG added was $0.5 \mathrm{wt} \%$, yielding a reduction by about $33.3 \%$ when compared to the pure PAO-6 ( 0.18). The friction coefficients for PAO-6 with different MG concentrations and $1 \mathrm{wt} \%$ dispersant were shown in Fig. 3(c). The variation trend of friction coefficient is consistent with the case in Fig. 3(b), and the lowest friction coefficient was 0.10 for PAO- 6 with $0.5 \mathrm{wt} \% \mathrm{MG}$ and $1 \mathrm{wt} \%$ dispersant. Figure 3(d) presents the friction coefficient curves of PAO-6, PAO-6 with MG (0.5 wt\%) and PAO-6 with MG (0.5 wt\%)/ 

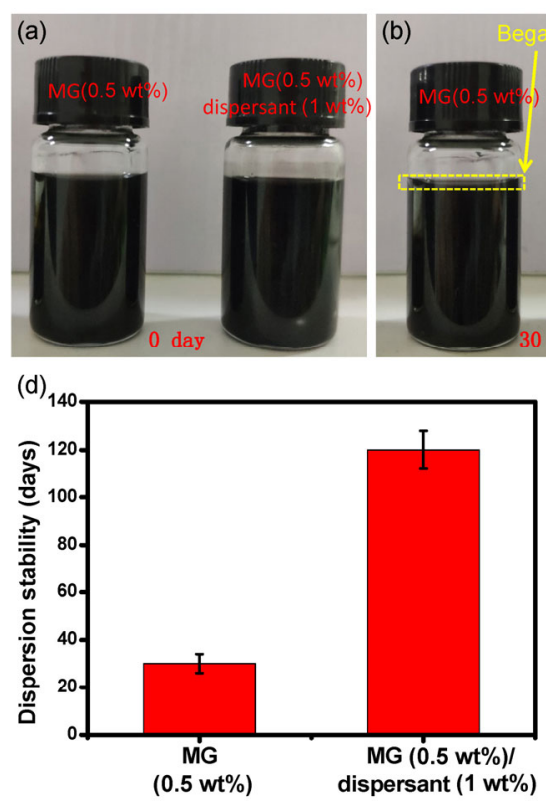
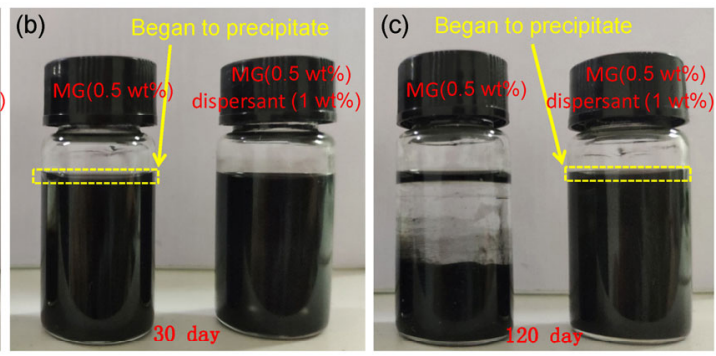

(e)

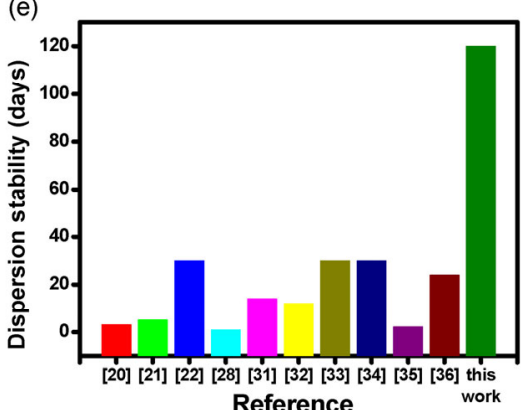

Fig. 2 (a-c) Photos of solution appearance in different time and (d) dispersion stability of MG $(0.5 \mathrm{wt} \%)$ and $\mathrm{MG}(0.5 \mathrm{wt} \%) / \mathrm{dispersant}$ (1 wt\%) in PAO-6. (e) The comparison of stable dispersion time for graphene as additives in oil from different literatures.

(a)
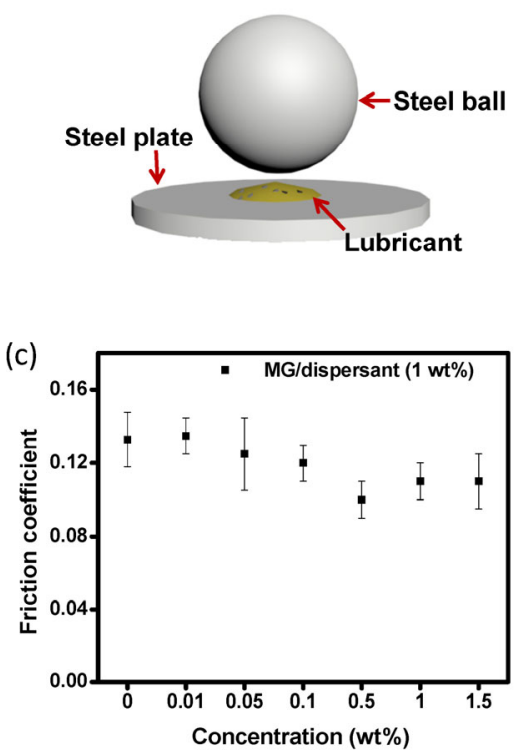
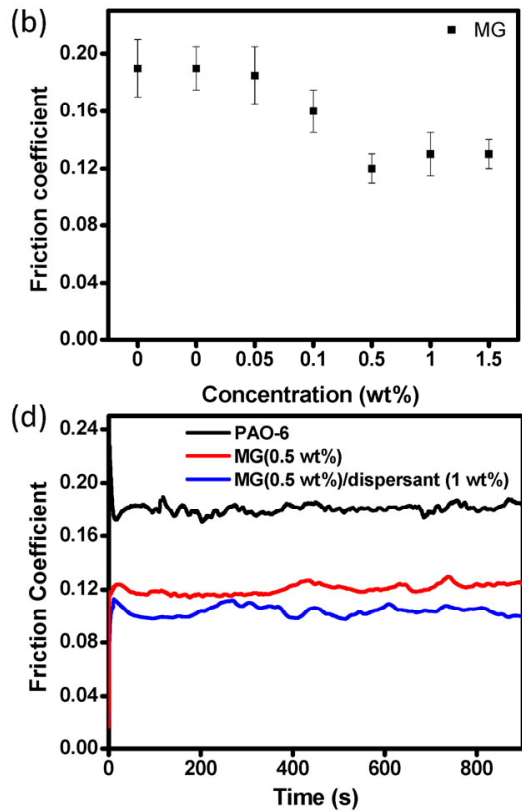

Fig. 3 (a) Schematic diagram of frictional experiments, the variation of friction coefficient with the change of concentration for (b) MG and (c) MG/dispersant, and (d) friction curves of PAO-6, MG (0.5 wt \%) and MG (0.5 wt \%)/dispersant (1 wt $\%)$.

dispersant (1 wt\%), respectively. For the base oil PAO-6, the friction coefficient increased in the beginning of the tests, then decreased and was kept stable at a relatively high value of about 0.18 . As for PAO-6 with MG (0.5 wt\%) and PAO-6 with MG (0.5 wt\%)/ dispersant $(1 \mathrm{wt} \%)$, it is obvious that the friction curves are stabilized at lower levels in the whole tests, which proved that the MG has good friction-reducing effect. Interestingly, the friction coefficient of PAO-6 with MG/dispersant is about 0.10 , which is $16.7 \%$ lower than that of PAO-6 with MG $(\sim 0.12)$. These results demonstrate that the MG dispersed in base oil has excellent capability of reducing friction.

In addition to the friction reduction, PAO- 6 with 
MG also provides excellent wear-resistance for the surfaces of tribo-pairs. Figures 4(a)-4(c) show the SEM images of wear tracks on steel plates for three different lubricants. The widths of wear scars were 119, 110, and $114 \mu \mathrm{m}$ for pure PAO-6, PAO-6 with MG (0.5 wt\%), and PAO-6 with MG (0.5 wt\%)/dispersant (1 wt \%), respectively. More details could be obtained from 3D surface morphologies (Figs. 4(d)-4(f)) and crosssectional profiles (Figs. 4(g)-4(i)). The wear track depths were $47 \mathrm{~nm}$ for PAO-6 with MG and $21 \mathrm{~nm}$ for PAO-6 with MG/dispersant, which were $79 \%$ and $90 \%$ lower than that of pure PAO-6 (221 nm), respectively. In a word, the use of MG and MG/dispersant in PAO-6 could prevent severe wear on the surface of steel plates during the sliding process.

Moreover, the tribological properties of commercial oils with MG and dispersant were also investigated. Figure 5(a) shows the friction coefficient curves of 10W-40 oil and 10W-40 oil with MG (0.5 wt\%)/ dispersant $(1 \mathrm{wt} \%)$ when the experimental time was 7,200 s. The friction coefficient of $10 \mathrm{~W}-40$ oil with MG/dispersant was about 0.10 , which was $9.1 \%$ lower than that of pure $10 \mathrm{~W}-40$ oil. Figure $5(\mathrm{~b})$ present the depth of wear tracks on the steel plates. It could be observed that the depth of wear tracks for 10W-40 oil with MG/dispersant was about $39 \mathrm{~nm}$, which was
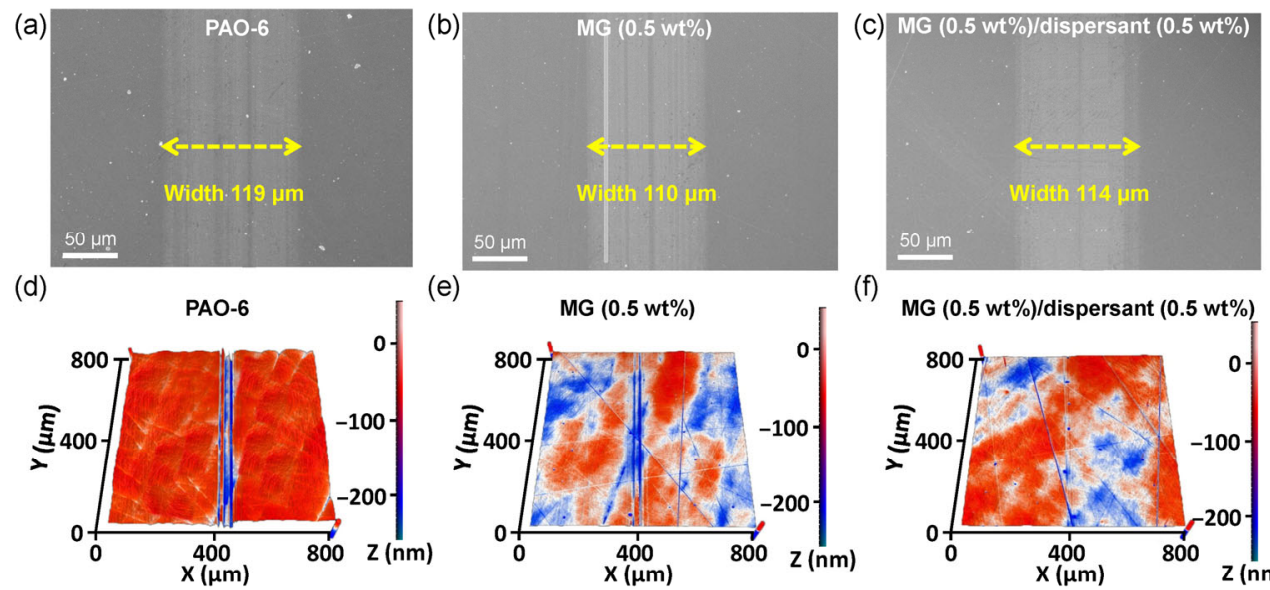

(e)

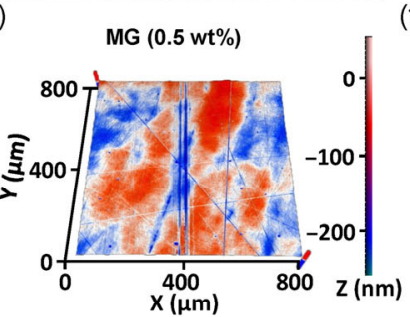

(f)
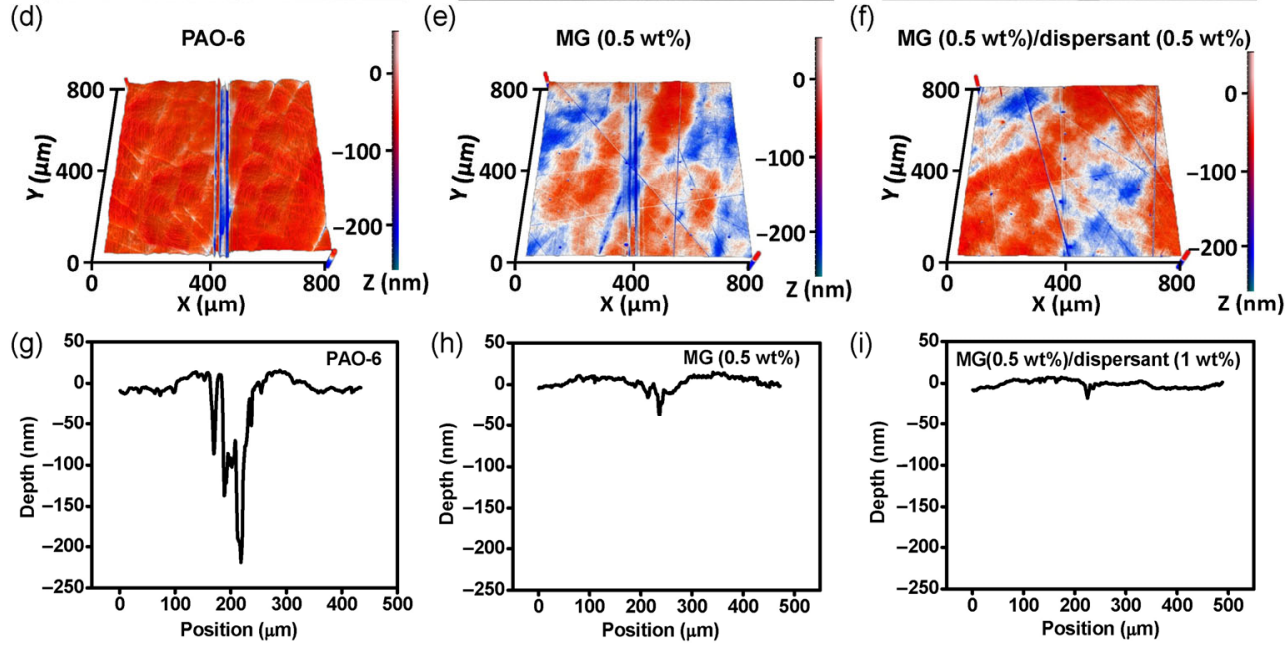

Fig. 4 Scanning electron microscope images, three-dimension surface images and the corresponding surface profiles of wear tracks for (a, d, g) PAO-6, (b, e, h) MG (0.5 wt \%), and (c, f, i) MG (0.5 wt\%)/dispersant (1 wt \%), respectively.
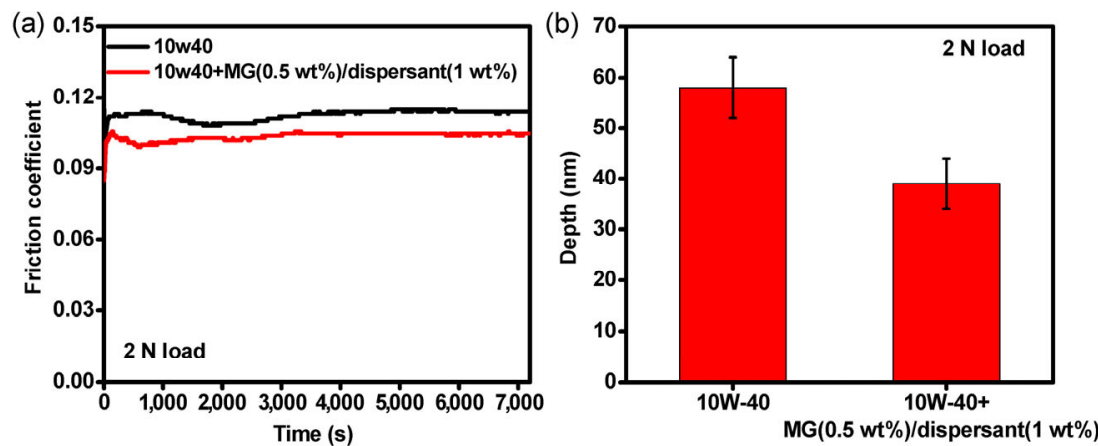

Fig. 5 (a) Friction coefficient curves of $10 \mathrm{~W}-40$ oil and $10 \mathrm{~W}-40$ oil with MG $(0.5 \mathrm{wt} \%) /$ dispersant (1 wt $\%)$ and (b) corresponding depth of wear tracks. 
$32.8 \%$ lower than that of pure $10 \mathrm{~W}-40$ oil. These results proved that MG with dispersant could also improve the tribological behaviors of commercial oil.

Furthermore, since Raman spectroscopy is an effective method for analyzing graphene [30], the lubrication mechanism was studied on the basis of Raman analysis of the wear tracks on steel plates. Figure 6 presents the Raman spectra of GO (Fig. 6(a)), MG before friction tests (Fig. 6(b)), wear track for PAO-6 with $0.5 \mathrm{wt} \%$ MG (Fig. 6(c)) and wear track for PAO-6 with $0.5 \mathrm{wt} \%$ MG and $1 \mathrm{wt} \%$ dispersant (Fig. 6(d)). In all the four cases, there are a D peak at $1,351 \mathrm{~cm}^{-1}$, a $G$ peak at $1,582 \mathrm{~cm}^{-1}$, a weak 2D peak at 2,702 $\mathrm{cm}^{-1}$, and a D $+\mathrm{D}^{\prime}$ peak at $3,081 \mathrm{~cm}^{-1}$. This demonstrates the formation of tribo-film in the contact area, and graphene is one of the components of the tribo-film. Besides, the intensity ratio of $\mathrm{D}$ and $\mathrm{G}$ peak of $\mathrm{MG}\left(I_{\mathrm{D}} / I_{\mathrm{G}}=1.08\right)$ is larger than that of pristine $\mathrm{GO}\left(I_{\mathrm{D}} / I_{\mathrm{G}}=0.90\right)$, which could be on account of the defects induced during the preparation process. After the friction tests, the $I_{\mathrm{D}} / I_{\mathrm{G}}$ ratios were 1.39 and 1.34 for PAO- 6 with $\mathrm{MG}$ and PAO-6 with MG/dispersant, respectively, suggesting that the mechanical action during the friction process could lead to an increase in the defects of graphene.

For more in-depth study of the tribological mechanism of PAO- 6 with MG as lubricant additive, the tribo-film formed on contact area of steel plate was further investigated through TEM observation. The
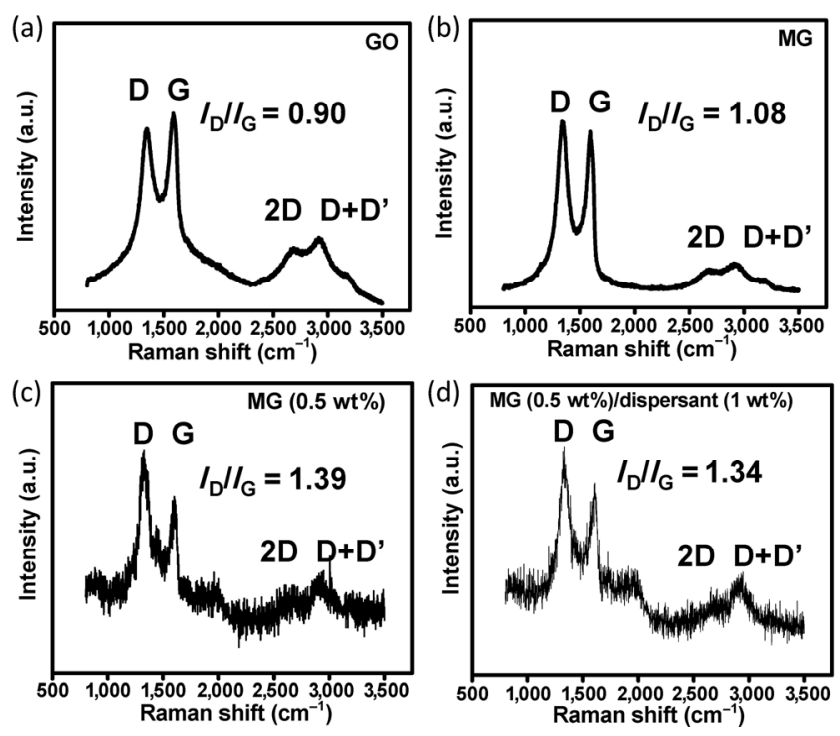

Fig. 6 Raman spectra of (a) GO, (b) MG, (c) wear track for PAO-6 with MG on steel plate and (d) PAO-6 with MG/dispersant on steel plate.
TEM-specimen of the tribo-film was prepared by focused ion beam (FIB) method from the contact area. Before the FIB process, the contact area was firstly coated by a protective metal layer of chromium through the ion sputtering technology. Afterwards, the sample was moved to the SEM-FIB chamber and another protective layer of platinum was deposited on the surface of $\mathrm{Cr}$ layer. Then the sample was incised and thinned using a $\mathrm{Ga}^{+}$ion source accelerated by a voltage of $30-5 \mathrm{kV}$ or $5 \mathrm{kV}$, and SEM images were delivered for positioning at the same time.

Figure 7(a) presents the TEM image of cross-sectional specimen obtained from the wear track for the PAO-6 with MG (0.5 wt\%)/dispersant (1 wt \%) under low magnification. It is easy to distinguish the steel substrate, the tribo-film, the $\mathrm{Cr}$ and the $\mathrm{Pt}$ protective layers. And the thickness of the tribo-film is about $90 \pm 10 \mathrm{~nm}$. Figures 7(b)-7(d) show high-resolution TEM images of three typical areas within the tribo-film. The graphene with stacked layer-like structure can be seen in Fig. 7(b), and the $d$-spacing value is about $0.498 \mathrm{~nm}$. Figure $7(\mathrm{c})$ shows the crystal lattice of $\mathrm{Fe}$, and the $d$-spacing value is about $0.204 \mathrm{~nm}$, corresponding to the (110) plane of Fe. Another crystal lattice structure with $d$-spacing value of about 0.252 is presented in Fig. 7(d), which is believed to be the (110) plane of $\mathrm{Fe}_{2} \mathrm{O}_{3}$. The $\mathrm{Fe}$ and $\mathrm{Fe}_{2} \mathrm{O}_{3}$ particles embedded in the tribo-film could be the wear debris of tribopairs formed during the sliding process. In addition, it can be observed that amorphous regions without an orderly structure exited within the tribofilm (Figs. 7(b)-7(d)), and several possible reasons might explain this phenomenon. Firstly, the preparation process of MG could lead to the amorphization of graphene. Secondly, the Raman pattern confirmed that friction tests would increase the defects in MG (Fig. 6(d)), which probably induced the partial amorphization of the crystal structure of graphene. Besides, the contamination and impurities introduced from preparation process of MG and friction tests might also be amorphous. Figures 7(e)-7(j) show the different elemental mappings of the TEM-specimen. $\mathrm{Cr}$ and $\mathrm{Pt}$ protective layers are presented in Figs. 7(f) and 7(g), and the tribo-film is mainly composed of C (Fig. 7(h)), Fe (Fig. 7(i)) and O (Fig. 7(j)).

In addition, low magnification TEM image of crosssectional specimen obtained from the wear track 
(a)

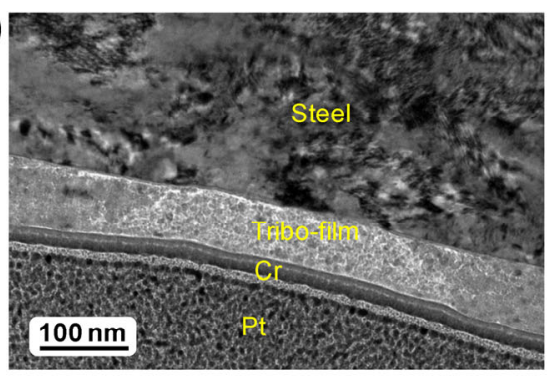

(c)

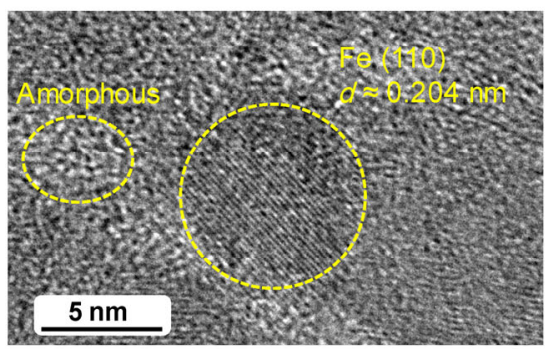

(b)

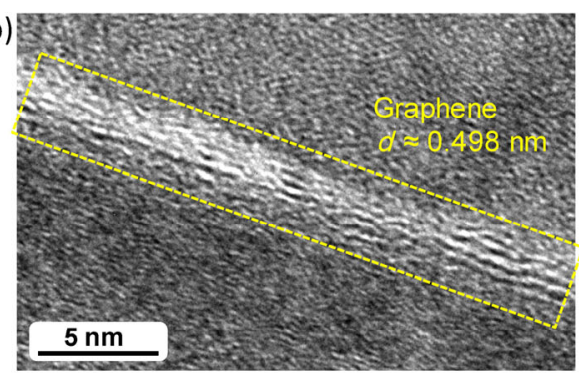

(d)

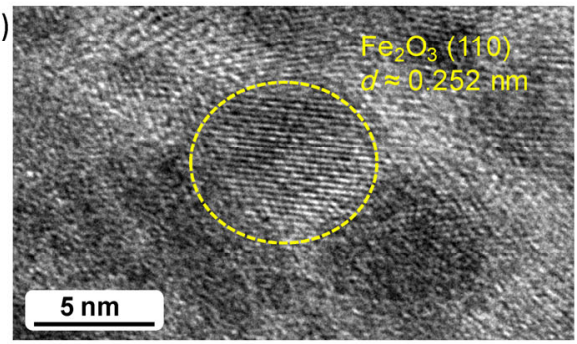

(e)

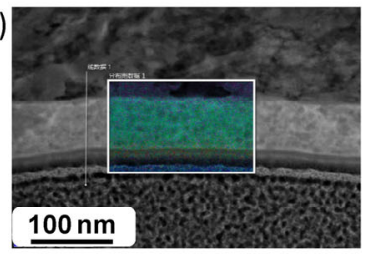

(h)

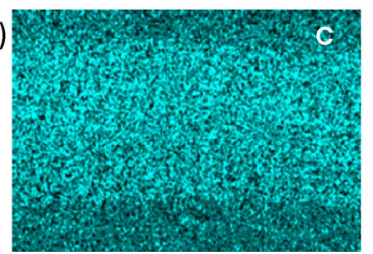

(f)

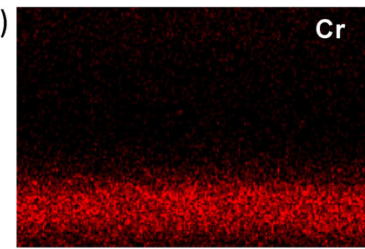

(i)

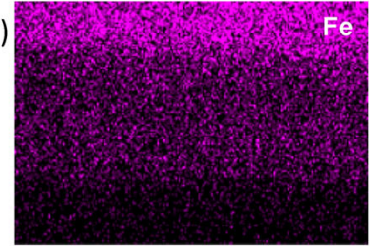

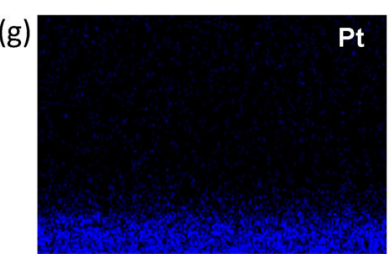

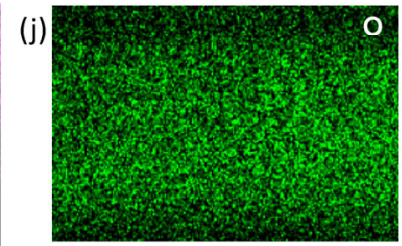

Fig. 7 (a) TEM image and (b-d) HR-TEM images of typical areas within the tribo-film formed on steel plate for PAO-6 with MG $(0.5 \mathrm{wt} \%) /$ dispersant $(1 \mathrm{wt} \%)$; (e-j) EDS-elemental mappings of the tribo-film.

for the PAO-6 with MG (0.5 wt\%) is presented in Fig. 8(a). The thickness of tribo-film is $100 \mathrm{~nm} \pm 5 \mathrm{~nm}$. Steel substrate, $\mathrm{Cr}$ and $\mathrm{Pt}$ layers could also be clearly found in the image. High-resolution TEM images regarding the detailed microstructures of the tribo-film are shown in Figs. 8(b)-8(d). The layered structure of graphene, the crystal structures of Fe ( $d$-spacing value $=$ $\sim 0.204 \mathrm{~nm})$ and $\mathrm{Fe}_{2} \mathrm{O}_{3}(d$-spacing value $=\sim 0.252 \mathrm{~nm})$ are quite similar to that of the tribo-film for PAO-6 with MG/dispersant. Besides, amorphous areas could also be found in the bulk of the tribo-film. Different EDS-elemental mappings of the TEM-specimen are presented in Figs. 8(e)-8(j). C (Fig. 8(h)), Fe (Fig. 8(i)) and $\mathrm{O}$ (Fig. 8(j)) are the main components of the tribo-film.

Graphene is a notable two-dimensional material which has been widely used as lubricating additive in both water-based and oil-based lubricants. And many studies have shown that tribo-film would form on the contact area during the friction test when graphene-containing lubricants were applied in the experimental process. Hiroshi et al. [18] investigated the frictional behaviors of GO nano-sheets as additives in water-based lubricants. Tribo-film was formed on the surfaces of tribopairs which was confirmed by SEM images and the corresponding elements mappings, thus enhancing the tribological performance of the lubricant. Researchers also added GO in hydrocarbon base oil to improve the lubricating performance, and tribo-film was detected in the wear tracks after friction process through Raman spectroscopy [31]. When reduced GO was employed as additive in base oil, the formation of tribo-film was proved by Raman spectroscopy and TEM images [14]. In our work, the analysis results of Raman spectra, TEM images and the 
(a)

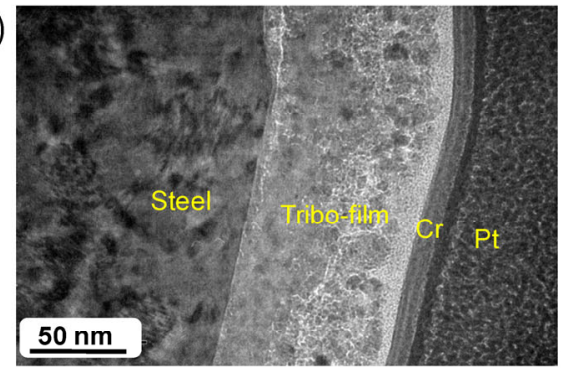

(c)

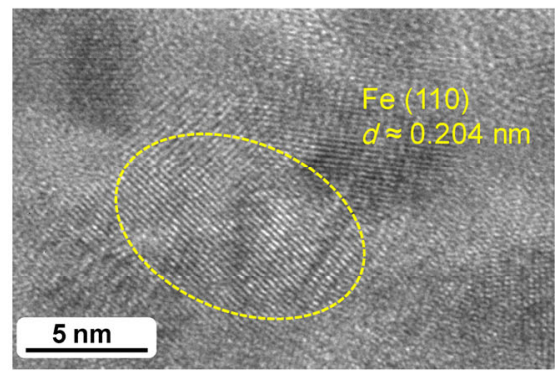

(e)

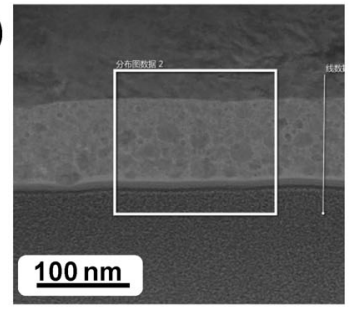

(h)

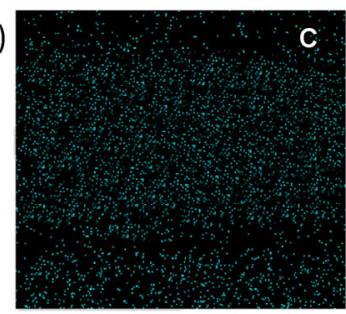

(f)

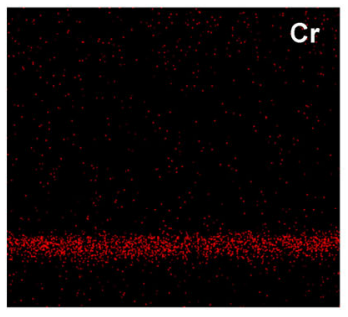

(i)

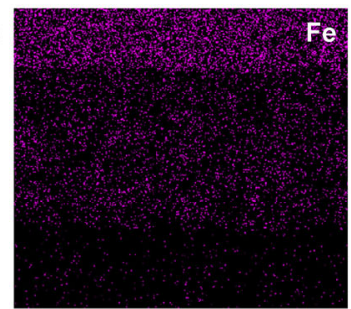

(b)

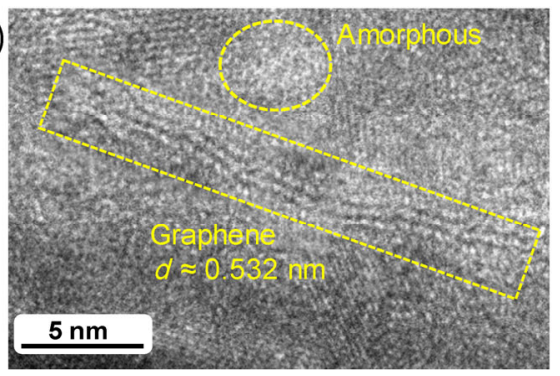

(d)

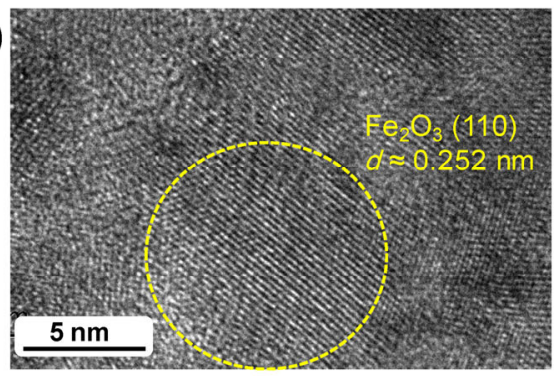

(g)
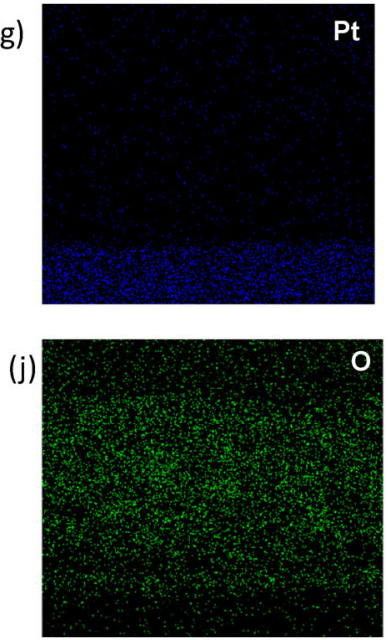

Fig. 8 (a) TEM image and (b-d) HR-TEM images of typical areas within the tribo-film formed on steel plate for PAO-6 with MG $(0.5 \mathrm{wt} \%)$; (e-j) EDS-elemental mappings of the tribo-film.

corresponding elemental mappings all demonstrate that the tribo-film existed in the wear tracks. It is believed that the tribo-film can prevent the direct contact of steel ball and steel plate, thus promoting the lubrication performances of the lubricant. In addition, graphene could easily enter into contact area during sliding process owing to its unique morphological structure. And shear resistance between the neighboring lamellae of graphene was quite low due to the weak inter-layer van der Waals interactions, which could also contribute to the improvement of tribological performance.

Interestingly, when MG (0.5 wt \%) and dispersant $(1 \mathrm{wt} \%$ ) were added into PAO-6 at the same time, not only the dispersion stability of graphene was improved, but also the tribological behaviors of the lubricant were enhanced as compared to PAO-6 with MG (0.5 wt\%). Firstly, the dispersant was mainly composed of polyisobutylene succinimide which contained nitrogen groups and long alkyl chains. The polar nitrogencontaining group would adsorb on the surface of the tribo-pairs while the long alkyl chain would form an alkyl chain shear layer (Fig. 9), thus enhancing the friction-reducing and anti-wear abilities of the lubricant [32, 33]. In addition, several studies have shown that the tribological properties of lubricants would also increase with the increase of dispersion stability of lubricating additives [34, 35]. 


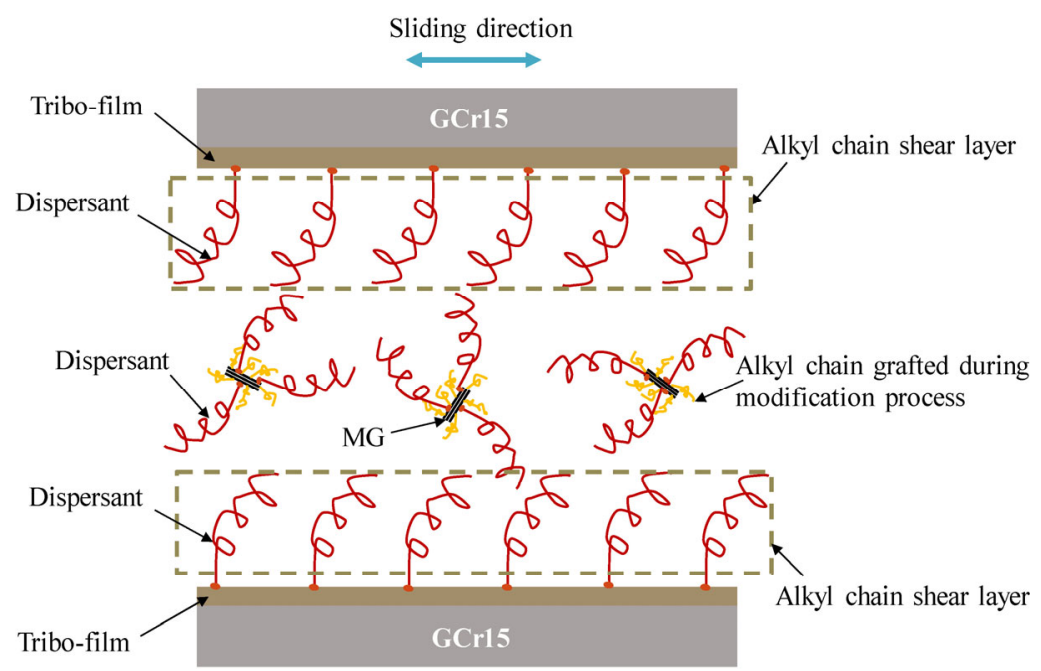

Fig. 9 Schematic diagram of the lubrication mechanism when PAO-6 with MG and dispersant was employed as lubricant.

In a word, we combined a chemical modification method with a unique dispersant to successfully increase the dispersion stability of graphene in base oil. In the meantime, the tribological performance of the oil with MG/dispersant was more superior to that of pure oil with MG and pure PAO-6. These results demonstrate that both the dispersion method of graphene in oil and the developed lubricant have great potential in engineering applications.

\section{Conclusions}

In summary, a novel dispersion method which combines chemical modification of graphene with a kind of effective dispersant has been successfully developed. Remarkable dispersibility of graphene as lubricating additive in PAO-6 is achieved with stable dispersion time up to about 120 days. Besides, the lubricant exhibits a significant improvement in tribological performance when compared to pure PAO-6. It is found that the combination of MG $(0.5 \mathrm{wt} \%)$ and dispersant $(1 \mathrm{wt} \%)$ has reduced friction coefficient and wear scar depth by about $40 \%$ and $90 \%$, respectively, with respect to the base oil. The excellent performances of MG and dispersant as lubricating additives in PAO-6 can be attributed to (a) the formation of tribo-film preventing the direct contact of tribopair surfaces, $(b)$ weak shear resistance between the lamellas of graphene, and (c) the lubrication effect of dispersant from the polyisobutylene succinimide.

\section{Acknowledgements}

The work is financially supported by the National Natural Science Foundation of China (Grant Nos. 51527901 and 51335005).

Electronic Supplementary Material: Supplementary material is available in the online version of this article at https://doi.org/10.1007/s40544-019-0359-2.

Open Access: This article is licensed under a Creative Commons Attribution 4.0 International License, which permits use, sharing, adaptation, distribution and reproduction in any medium or format, as long as you give appropriate credit to the original author(s) and the source, provide a link to the Creative Commons licence, and indicate if changes were made.

The images or other third party material in this article are included in the article's Creative Commons licence, unless indicated otherwise in a credit line to the material. If material is not included in the article's Creative Commons licence and your intended use is not permitted by statutory regulation or exceeds the permitted use, you will need to obtain permission directly from the copyright holder.

To view a copy of this licence, visit http://creativecommons.org/licenses/by/4.0/.

\section{References}

[1] Lee C, Wei X D, Kysar J W, Hone J. Measurement of the 
elastic properties and intrinsic strength of monolayer graphene. Science 321(5887): 385-388 (2008)

[2] Stankovich S, Dikin D A, Dommett G H B, Kohlhaas K M, Zimney E J, Stach E A, Piner R D, Nguyen S T, Ruoff R S. Graphene-based composite materials. Nature 442(7100): 282-286 (2006)

[3] Li X M, Xie D, Park H, Zeng T H, Wang K L, Wei J Q, Zhong M L, Wu D H, Kong J, Zhu H W. Anomalous behaviors of graphene transparent conductors in graphene-silicon heterojunction solar cells. Adv Energy Mater 3(8): 1029-1034 (2013)

[4] Gu W T, Zhang W, Li X M, Zhu H W, Wei J Q, Li Z, Shu Q K, Wang C, Wang K L, Shen W C, et al. Graphene sheets from worm-like exfoliated graphite. J Mater Chem 19(21): 3367-3369 (2009)

[5] Bunch J S, Verbridge S S, Alden J S, Van Der Zande A M, Parpia J M, Craighead H G, McEuen P L. Impermeable atomic membranes from graphene sheets. Nano Lett 8(8): 2458-2462 (2008)

[6] Matsumura K, Chiashi S, Maruyama S, Choi J. Macroscale tribological properties of fluorinated graphene. Appl Surf Sci 432: 190-195 (2018)

[7] Huang Y H, Yao Q Z, Qi Y Z, Cheng Y, Wang H T, Li Q Y, Meng Y G. Wear evolution of monolayer graphene at the macroscale. Carbon 115: 600-607 (2017)

[8] Shi Z, Shum P, Wasy A, Zhou Z F, Li L K Y. Tribological performance of few layer graphene on textured M2 steel surfaces. Surf Coat Technol 296: 164-170 (2016)

[9] Kim H J, Shin D G, Kim D. Frictional behavior between silicon and steel coated with graphene oxide in dry sliding and water lubrication conditions. Int J Prec Eng Manuf-Green Technol 3(1): 91-97 (2016)

[10] Banerji A, Bhowmick S, Lukitsch M J, Alpas A T. Friction behaviour of multilayered graphene against steel. MRS Online Proc Libr Arch 1812: 1-8 (2016)

[11] Holmberg K, Erdemir A. Influence of tribology on global energy consumption, costs and emissions. Friction 5(3): 263-284 (2017)

[12] Wu P, Chen X C, Zhang C H, Luo J B. Synergistic tribological behaviors of graphene oxide and nanodiamond as lubricating additives in water. Tribol Int 132: 177-184 (2019)

[13] Wu L P, Gu L, Xie Z J, Zhang C W, Song B Y. Improved tribological properties of $\mathrm{Si}_{3} \mathrm{~N}_{4} / \mathrm{GCr} 15$ sliding pairs with few layer graphene as oil additives. Ceram Int 43(16): 1421814224 (2017)

[14] Ismail N A, Bagheri S. Highly oil-dispersed functionalized reduced graphene oxide nanosheets as lube oil friction modifier. Mater Sci Eng B 222: 34-42 (2017)

[15] Liu Y H, Wang X K, Pan G S, Luo J B. A comparative study between graphene oxide and diamond nanoparticles as water-based lubricating additives. Sci China Technol Sci 56(1): 152-157 (2013)

[16] Li Y R, Zhao J, Tang C, He Y Y, Wang Y F, Chen J Y, Zhou Q Q, Wang B Y, Wei F, Luo J B, et al. Highly exfoliated reduced graphite oxide powders as efficient lubricant oil additives. Adv Mater Interfaces 3(22): 1600700 (2016)

[17] Dou X, Koltonow A R, He X L, Jang H D, Wang Q, Chung Y, Huang G X. Self-dispersed crumpled graphene balls in oil for friction and wear reduction. Proc Natl Acad Sci USA 113(6): 1528-1533 (2016)

[18] Kinoshita H, Nishina Y, Alias A A, Fujii M. Tribological properties of monolayer graphene oxide sheets as water-based lubricant additives. Carbon 66: 720-723 (2014)

[19] Lin J S, Wang L W, Chen G H. Modification of graphene platelets and their tribological properties as a lubricant additive. Tribol Lett 41: 209-215 (2011)

[20] Cai Z B, Zhao L, Zhang X, Yue W, Zhu M H. Combined effect of textured patterns and graphene flake additives on tribological behavior under boundary lubrication. PLoS One 11(4): e0152143 (2016)

[21] Hirata M, Gotou T, Horiuchi S, Fujiwara M, Ohba M. Thin-film particles of graphite oxide 1: High-yield synthesis and flexibility of the particles. Carbon 42(14): 2929-2937 (2004)

[22] Zhang Y B, Liu L Q, Sun B, Wang G R, Zhang Z. Preparation of lipophilic graphene oxide derivates via a concise route and its mechanical reinforcement in thermoplastic polyurethane. Compos Sci Technol 134: 36-42 (2016)

[23] Guo Y B, Zhang S W. The tribological properties of multilayered graphene as additives of PAO2 oil in steel—steel contacts. Lubricants 4(3): 30 (2016)

[24] Song H J, Wang Z Q, Yang J. Tribological properties of graphene oxide and carbon spheres as lubricating additives. Appl Phys A 122(10): 933 (2016)

[25] Mungse H P, Kumar N, Khatri O P. Synthesis, dispersion and lubrication potential of basal plane functionalized alkylated graphene nanosheets. RSC Adv 5(32): 25565-25571 (2015)

[26] Mungse H P, Khatri O P. Chemically functionalized reduced graphene oxide as a novel material for reduction of friction and wear. J Phys Chem C 118(26): 14394-14402 (2014)

[27] Zhao J, Li Y R, Wang Y F, Mao J Y, He Y Y, Luo J B. Mild thermal reduction of graphene oxide as a lubrication additive for friction and wear reduction. $R S C A d v$ 7(3): 1766-1770 (2017)

[28] Zhang L, He Y, Zhu L, Yang C, Niu Q H, An C L. In situ alkylated graphene as oil dispersible additive for friction and wear reduction. Ind Eng Chem Res 56(32): 9029-9034 (2017) 
[29] Tessonnier J P, Barteau M A. Dispersion of alkyl-chainfunctionalized reduced graphene oxide sheets in nonpolar solvents. Langmuir 28(16): 6691-6697 (2012)

[30] Ferrari A C, Meyer J C, Scardaci V, Casiraghi C, Lazzeri M, Mauri F, Piscanec S, Jiang D, Novoselov K S, Roth S, et al. Raman spectrum of graphene and graphene layers. Phys Rev Lett 97(18): 187401 (2006)

[31] Chen Z, Liu Y H, Luo J B. Tribological properties of few-layer graphene oxide sheets as oil-based lubricant additives. Chin $J$ Mech Eng 29(2): 439-444 (2016)

[32] Yang W Q, Chen B S, Fang J H, Wang J, Gu K C. Tribological-Synergistic effect of group containing nitrogen and boric acid ester from methyl oleate. Acta Pet Sin Pet Proc Sect 32(2): 305-311 (2016)

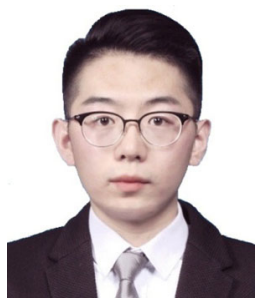

Pu WU. He received his bachelor degree in mechanical engineering in 2014 from Tsinghua University, Beijng, China. After then, he was a Ph.D. student in the State Key Laboratory of Tribology at the same

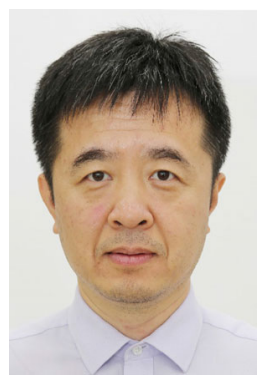

Chenhui ZHANG. He received his Ph.D. degree in mechanical engineering from Tsinghua University, Beijing, China in 2004. From then, he worked at the State Key Laboratoty of Tribology at Tsinghua. From February 2011 to August 2011, he was invited to Luleå University

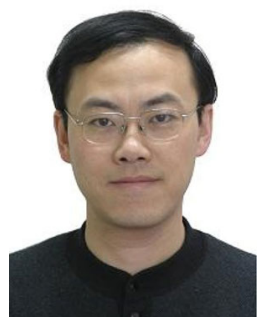

Jiyang ZHANG. He received his B.S., M.S., and Ph.D. degrees from the Beihang University, Beijing, China, in 1992, 1999, and 2015, respectively.
[33] Tariq M, Serro A P, Colaço R, Saramago B, Lopes J N C, Rebelo L P N. Effect of alkyl chain length on the adsorption and frictional behaviour of 1-alkyl-3-methylimidazolium chloride ionic liquid surfactants on gold surfaces. Colloids Surf A Phys Eng Aspects 377(1-3): 361-366 (2011)

[34] Yi S L, Yu G X, Zhou X L, Jin Y Q, Li C L. Effect of dispersivity and stability of $\mathrm{LaF}_{3}$ nanoparticles in lubricating oils on their tribological properties. J East China Univ Sci Technol Nat Sci Ed 32(12): 1392-1395 (2006)

[35] Gulzar M, Masjuki H H, Kalam M A, Varman M, Zulkifli N W M, Mufti R A, Zahid R. Tribological performance of nanoparticles as lubricating oil additives. J Nanopart Res 18(8): 223 (2016)

university. He has recently obtained his Ph.D. degree in mechanical engineering at Tsinghua University in 2019. His research interests include tribological properties of graphene and tribometer with high vacuum and low temperature.

of Technology in Sweden as a visiting scholar. Then he was invited to Weizmann Institute of Science in Israel as a visiting scientist from February 2012 to January 2013. His current position is a professor at Tsinghua University. His research areas cover surface coatings technology and lubrication theory. His current research interest focuses on the superlubricity.

He is currently a senior researcher at Beijing Institute of Control Engineering. His research areas cover the spacecraft actuator design and the application of superlubricity. 